\title{
Vorsorgen oder Ausbrennen
}

\section{Der Körper des Werktätigen und der „energetische Imperativ“ in der Weimarer Republik}

Vorsorgekonzepte an die Kategorie Körper heranzutragen, scheint zunächst auf besonders basale, ja archaische Verhaltensweisen abzuzielen. In diesem Sinne ist die Körper-Vorsorge vielleicht die ursprünglichste Form der Vorsorge überhaupt: wärmende Felle beschaffen, um den Körper vor Kälte zu schützen, den Magen so weit wie möglich füllen, um eine Zeit ohne Nahrung zu überstehen, einen Panzer anlegen, um im Kampf mit Tier und Mensch nicht verwundet zu werden.

Seit der Antike wird neben den Maßnahmen zur Vermeidung von Kälte, Hunger und Verwundung die Sorge um die Erhaltung der Gesundheit in den Quellen greifbar. Die Diätetik entwickelte Vorschriften für eine Lebensführung, die der Entstehung von Krankheiten vorbeugte. ${ }^{1}$ An ihre Seite traten Gebete zu den Göttern, die - wie auf alle Zukunft - auch auf die künftige Gesundheit Einfluss nahmen. Das christliche Mittelalter akzentuierte diesen religiösen Bezug noch, indem es Krankheit zu einer Strafe Gottes erklärte. So meinte Vorsorge vor allem die Vermeidung von Sünden. Erst die Aufklärungsbewegung des 18. Jahrhunderts rückte die Frage von Gesundheit und Krankheit wieder vorrangig in die materielle - und nicht nur moralisch-religiöse Verantwortung des Individuums. Der vernunftbegabte Einzelne, der um die Verwurzelung seiner Existenz in der Natur wusste, sollte seinen Körper so behandeln, dass er dauerhaft leistungs- und genussfähig blieb, anstatt Krankheit und Siechtum anheimzufallen. $^{2}$

Wichtigstes Mittel hierzu war die Hygiene, die alle äußeren Einflüsse auf den Körper und all seine inneren Triebkräfte daraufhin befragte, welchen Nutzen und Schaden sie zeitigen könnten. Vor allem bürgerliche Schichten verschrieben sich dieser Doktrin. Schädliche äußere Einflüsse waren durch allgemeine Reinlichkeit, Körperpflege und Achtsamkeit bei Wasser und Nahrungsmitteln auszuschließen. ${ }^{3}$ Gegen die Ansteckung mit Krankheitserregern wirkten Schutzimpfungen bei möglichst großen Teilen der Bevölkerung, für die Staat und Militär zuständig wurden. ${ }^{4}$

1 Michel Foucault, Ästhetik der Existenz. Schriften zur Lebenskunst, Frankfurt a. M. 2007.

2 Philipp Sarasin, Reizbare Maschinen. Eine Geschichte des Körpers 1765-1914, Frankfurt a. M. 2001, S. 17-19.

3 Ebenda, S. 118-136 und 272-288.

4 Ute Frevert, Krankheit als politisches Problem 1770-1880. Soziale Unterschichten in Preußen zwischen medizinischer Polizei und staatlicher Sozialversicherung, Göttingen 1984; Jürgen Osterhammel, Die Verwandlung der Welt. Eine Geschichte des 19. Jahrhunderts, München 2011, S. 268-277; für die Zeit nach 1871 auch Malte Thießen, Vom immunisierten Volkskörper zum „präventiven Selbst“. 
Bei den Triebkräften galt das Gebot des maßvollen Auslebens: Ihre Unterdrückung war schädlich, weil sie zu nervösen Störungen führte, ihre exzessive Befriedigung indes ebenso, weil sie Körper und Geist zu zerrütten drohte. ${ }^{5}$ Der durch die Hygiene aufgeklärte Mensch orientierte sich an einem als „natürlich“ begriffenen Rhythmus. Er ließ sich nicht - weder im Sinne der fanatischen Abwehr, noch des haltlosen Zulassens - von seinen Trieben beherrschen, sondern gestaltete den Umgang mit ihnen nach Maßgabe souveräner Willensentscheidungen. Diese Haltung gehört zu den Leitbildern der Moderne, die bis heute nachwirken.

Bringt man die Körper-Vorsorge hingegen mit dem Begriff des Ressourcenmanagements in Verbindung, so ist dies zunächst überraschend. Von Ressourcenmanagement ist normalerweise dann die Rede, wenn es darum geht, begrenzte Vorräte vor der Übernutzung zu schützen; an diesen Raubbau zu treiben, hieße, sie künftig nicht mehr zur Verfügung zu haben. Auf zwei Feldern kann das Ressourcenmanagement folglich als besonders wichtiger Modus der Vorsorge gelten: auf dem Feld der Bewirtschaftung von Rohstoffen, deren Vorräte in absehbarer Zeit erschöpft sein werden, und beim Umgang mit natürlichen Hilfsmitteln wie Trinkwasser, die nur in begrenztem Umfang zur Verfügung stehen. Es sind also ökonomische und ökologische Kontexte, in denen sich der Terminus Ressourcenmanagement etabliert hat. Bezüge zum Körper scheinen sich demgegenüber nur in indirekter und vermittelter Form konstruieren $\mathrm{zu}$ lassen.

\section{Vorsorgen in der industriellen Moderne: Zur Formierung des Arbeiterkörpers}

Anders stellt sich die Situation dar, wenn der Körper nicht als eine Größe an sich, sondern in einem bestimmten modus operandi aufgefasst wird: als arbeitender Körper, noch konkreter: als industriellen Arbeitsprozessen ausgesetzter Körper. In dieser Perspektive lässt sich einer „Körpergeschichte der Industrie“ nachspüren bzw. einer „Körpergeschichte der Arbeit an Maschinen“, welche die menschliche Physis besonderen Anforderungen aussetzt. ${ }^{6}$ Mit diesem Ansatz werden grundsätzliche Fragen aufgeworfen: Welche Folgen hatte die Arbeit an Maschinen für die menschliche Physis und zwar nicht nur im Sinne der Unfallverhütung, die von der Geschichtsschreibung zu den großen Sozialversicherungen bereits abgehandelt worden ist, sondern im Hin-

Impfen als Biopolitik und soziale Praxis vom Kaiserreich zur Bundesrepublik, in: Vierteljahrshefte für Zeitgeschichte 61 (2013), S. 35-64.

5 Sarasin, Reizbare Maschinen, S. 356-433.

6 Siehe zu diesem Ansatz auch die Beiträge in Body Politics. Zeitschrift für Körpergeschichte 1 (2013), H. 1, hrsg. von Peter-Paul Bänziger: Fordismus. 
blick auf den Wandel des allgemeinen Körperverständnisses oder auf Maßnahmen zur Vorsorge und Vorbereitung auf die Anforderungen der industriellen Welt?

Um von der Körpergeschichte der Industrie zu den Ressourcen und zum Ressourcenmanagement zu kommen, ist jedoch noch ein erläuternder Zwischenschritt nötig. Er bezieht sich auf die menschliche Arbeitskraft bzw. auf das Zusammenspiel von Arbeitsfähigkeit und Ermüdung. Dieses Zusammenspiel wurde seit dem späten 19. Jahrhundert neu definiert. Bis dahin hatte die Überzeugung vorgeherrscht, die Arbeitsleistung eines Menschen hänge im Wesentlichen von seiner Willenskraft ab. Wenn sich Ermüdung einstelle, gelte es, gegen die körperliche Schwäche anzukämpfen. Wenn ein Arbeiter der Müdigkeit nachgebe, sei dies vor allem auf moralische Schwäche zurückzuführen. Mit dem Durchbruch der Thermodynamik und ihren Erkenntnissen zur Physik der Energie entstand ein völlig neues Bild. Da sich Energie, wenn sie zur Verrichtung von Arbeit genutzt wird, zwangsläufig reduziert, erfolgt die Ermüdung des Arbeiters mit der Folgerichtigkeit eines Naturgesetzes - der Appell an die Willenskraft ist nur begrenzt wirksam. Stattdessen erschien der Arbeiterkörper nun als dynamisches System von Prozessen der Energieumwandlung. Energie floss ab und musste wieder hinzugefügt, also durch Regeneration neu aufgebaut werden. ${ }^{7}$ Leistung und Ermüdung verwandelten sich damit von einem moralischen zu einem wissenschaftlichen Problem. Je besser der Körper des Arbeiters verstanden, je besser sein Stoffwechsel analysiert wurde, desto besser konnte seine - als Ressource begriffene - Arbeitskraft bewirtschaftet werden. Ressourcenmanagement bedeutete also, die Arbeitskraft vor der Übernutzung zu schützen, mithin vorzusorgen, dass dem Arbeiterkörper ein hinreichendes Maß an Energie zur Verfügung stand und die strukturelle Ermüdung verhindert wurde.

Kurz vor dem Ersten Weltkrieg war in Deutschland eine Wissenschaft entstanden, die sich gezielt solchen Problemen widmete. Das neue Fach hieß Arbeitsphysiologie und verstand sich als ein Teilgebiet der Arbeitswissenschaften. Seine Protagonisten waren Mediziner, die als Physiologen ausgebildet waren und Stoffwechselprozesse unter den Bedingungen körperlicher Anstrengung untersuchten. Sie wollten den Abund Aufbau von Energie im Körper des arbeitenden Menschen wissenschaftlich exakt beschreibbar machen.1913 wurde der neuen Disziplin durch die Gründung eines „Kaiser-Wilhelm-Instituts für Arbeitsphysiologie“ (KWIfA) in Berlin offizielle Anerkennung gezollt. Erster Direktor des Hauses wurde Maximilian Rubner (1854-1932), Medizinprofessor an der Berliner Universität. Ihm folgte 1926 sein Schüler Edgar Atzler (1887-1938) nach. ${ }^{8}$

7 Anson Rabinbach, Ermüdung, Energie und der menschliche Motor, in: Philipp Sarasin/Jakob Tanner (Hrsg.), Physiologie und industrielle Gesellschaft. Studien zur Verwissenschaftlichung des Körpers im 19. und 20. Jahrhundert, Frankfurt a. M. 1998, S. 286-312, hier S. 297.

8 Rüdiger Hachtmann, Ein Kind der Ruhrindustrie? Die Geschichte des Kaiser-Wilhelm-Instituts für Arbeitsphysiologie von 1913 bis 1945, in: Westfälische Forschungen 60 (2010), S. 73-154, hier S. 77-79. 
1921 leitete das KWIfA eine Kooperation mit einer anderen Institution des Berliner Wissenschaftsbetriebes ein, die sich ähnlichen Fragestellungen verschrieben hatte: der Deutschen Hochschule für Leibesübungen, die 1920 als eine der ersten Sporthochschulen der Welt gegründet worden war. An ihr fand jene junge Sportwissenschaft ihren Platz, zu deren wichtigsten Anliegen die Analyse von Leistungserbringung und Ermüdung beim Sport treibenden Menschen gehörte. Der Unterschied zu den Arbeitswissenschaften bestand nur darin, dass der Anlass für die körperliche Anstrengung ein anderer war - hier der Sport, da die Arbeit. Die Auswirkungen dieser Anstrengung auf den Stoffwechsel waren indes für beide Disziplinen zentraler Gegenstand. ${ }^{9}$ Auch deshalb sah der Kooperationsvertrag zwischen den beiden Häusern die wechselseitige Benutzung der Labors vor. ${ }^{10}$ Dort wurden Messungen am Menschen unter den Bedingungen von physischer Belastung vorgenommen. Die Probanden waren in der Regel Studierende der Sporthochschule. Sie vollzogen Bewegungen, die für sportliche Übungen und/oder Arbeitsvorgänge charakteristisch waren. Spezielle Geräte, sogenannte Respirationsapparate, maßen den dabei anfallenden Energieverbrauch. Durch leichte Variationen dieser Bewegungen und wiederholte Messungen wurde ermittelt, bei welchem Bewegungstyp der Energieaufwand am geringsten war. Davon profitierten die Sportler unmittelbar, aber auch für die Industrie ließen sich Empfehlungen herleiten, welche Bewegungsschablonen den Arbeitern künftig vorzugeben seien. ${ }^{11}$ In diesem Verständnis dienten ökonomische Bewegungsabläufe der Vorsorge vor der Verschwendung von Kraftressourcen.

Die deutsche Industrie befand sich seit dem Ersten Weltkrieg in einem Umbruch, der durch die Rezeption tayloristischer und fordistischer Methoden geprägt war und mit einer forcierten Verwissenschaftlichung des Produktionsprozesses einherging. Die Analyse von Arbeitsvorgängen stand also ohnehin auf der Agenda, auch wenn Taylor und Ford vor allem auf die Vermeidung unnötiger Wege und Bewegungen abgezielt hatten; eingemündet waren diese Überlegungen bekanntlich in das System der Fließbandproduktion. Die deutschen Arbeitsphysiologen übernahmen viele Grundgedanken der amerikanischen Industriepioniere, bestanden aber darauf, dass ihre Vorgehensweise auf einem anderen Prinzip beruhte. Taylor und Ford sei es nur darum gegangen, die Effizienz der Arbeit zu erhöhen und damit die Produktivität zu steigern; was mit den Arbeitern dabei geschah, sei unerheblich gewesen. Brannten sie

9 Frank Becker, Rationalisierung - Körperkultur - Neuer Mensch. Arbeitsphysiologie und Sport in der Weimarer Republik, in: Theo Plesser/Hans-Ulrich Thamer (Hrsg.), Arbeit, Leistung und Ernährung. Vom Kaiser-Wilhelm-Institut für Arbeitsphysiologie in Berlin zum Max-Planck-Institut für molekulare Physiologie und Leibniz Institut für Arbeitsforschung in Dortmund, Stuttgart 2012, S. 149-170, hier S. 154.

10 Noyan Dinçkal, „Sport ist die körperliche und seelische Selbsthygiene des arbeitenden Volkes“: Arbeit, Leibesübungen und Rationalisierungskultur in der Weimarer Republik, in: Body Politics 1 (2013), S. 71-97, hier S. 82.

11 Becker, Rationalisierung, S. 155. 
aus, wurden sie durch frische Kräfte ersetzt. In den USA habe man sich einen solchen „Menschenverschleiß“ leisten können, da die Einwanderung ständig für Kompensation sorgte. In Deutschland jedoch, das durch den Ersten Weltkrieg und die Gebietsabtretungen ohnehin hohe Bevölkerungsverluste habe hinnehmen müssen, sei ein schonender Umgang mit Humanressourcen geboten. ${ }^{12}$ Vorsorge als Ressourcenschonung stand in Deutschland also auch im Kontext des zeitgenössischen Krisendiskurses.

So diente die Energiemessung, wie sie die Arbeitsphysiologen gemeinsam mit den Sportwissenschaftlern betrieben, keineswegs der Reduktion des Energieaufwandes an der einen Stelle, um dem Arbeiter zusätzlichen Energieaufwand an anderer Stelle aufzubürden. Entscheidend sei vielmehr, so wurde argumentiert, das Auffinden des am wenigsten energieaufwändigen Weges zum Arbeitsergebnis, das anschließend aber nicht in die Höhe geschraubt werden dürfe; die eingesparte Energie solle für den Arbeiter ein wirklicher Gewinn sein. Atzler betrieb in diesem Zusammenhang Begriffspolitik, indem er im Hinblick auf Taylor und Ford von einer „Maximierung“ der Arbeitsleistung sprach, für sein Konzept hingegen den Terminus der „Optimierung“ in Anspruch nahm: Optimal war demnach eine möglichst hohe Produktivität bei möglichst niedrigem Energieaufwand. ${ }^{13}$

Zum Management des Energiehaushalts gehörte es aber auch, die Wiederherstellung verbrauchter Energie zu ermöglichen. Dies lenkte den Blick auf die Arbeitspausen des Arbeiters im Werk und auf seine Freizeitaktivitäten jenseits der Werkstore. Wieder bot sich eine Kooperation mit der Sportwissenschaft an. Deren Vertreter waren der Ansicht, bei Ermüdung sei nicht, jedenfalls nicht dauerhaft, die schlichte Untätigkeit, das Ausruhen, geboten, sondern eine neue Form der Aktivität, welche die negativen Folgen der vorherigen Anstrengung ausgleiche. So wurden Formen der Pausengymnastik entwickelt, die die überbeanspruchte Muskulatur entlasten, Folgeschäden der einseitigen Belastung vorbeugen und durch die Anregung von Kraftzentren im Körper für neue Frische sorgen sollten. Für den Bereich der Freizeit wurden diese Überlegungen noch ausgeweitet: Als regenerativ galten hier Formen der körperlichen Betätigung, die nicht erzwungen, sondern freiwillig ausgeführt wurden. Wer, wie es beim Sport der Fall sei, aus Freude an der Bewegung und am Spiel seine Kräfte übe, erhöhe letztlich die Spannkraft seiner Physis. Zu vermeiden sei nur eine Überanstrengung, die den Werktätigen am Montagmorgen ausgelaugt zur Arbeit zurückkehren lasse. Die schädlichsten Folgen habe aber gewiss der übermäßige Konsum von

12 Michael Hau, Biopolitik der Leistungssteigerung: Arbeit als Sport in der Weimarer Republik und im Nationalsozialismus, in: Limbus. Australisches Jahrbuch für germanistische Literatur- und Kulturwissenschaft 2 (2009), S. 87-101, hier S. 90.

13 Hachtmann, Kind, S. 80. 
Alkohol und Nikotin, der das Freizeitverhalten vieler Arbeiter in der Vergangenheit gekennzeichnet habe. ${ }^{14}$

Ein weiterer Aspekt des Humanressourcen-Managements war die passende Zuordnung von Arbeitern und Arbeitsplätzen. Anders formuliert: Jeder Arbeiter sollte am richtigen Platz stehen. Die körperlichen Voraussetzungen, die eine Person mitbrachte, ließen sie für bestimmte Arbeitsvorgänge besonders geeignet erscheinen. Andere Arbeitsvorgänge, für die ihre Physis nicht ausgelegt war, führten dagegen zu einem schnellen Kräfteverschleiß. Ein Arbeiter, der an einem Arbeitsplatz A nach kurzer Zeit „ausbrannte“, konnte an einem Arbeitsplatz B dauerhaft gute Leistungen bringen. Es sei ein Gebot der volkswirtschaftlichen Vernunft, durch Eignungstests dafür zu sorgen, Schulabgänger von vornherein den passenden Berufen zuzuführen. Expertise für die Durchführung solcher Tests stellten die Sportwissenschaftler bereit, die dieselben Methoden einsetzten, um jungen Sportinteressierten die Sportart zu empfehlen, in der sie voraussichtlich die besten Resultate erzielen würden. Am Horizont erschien die Vision von Reihenuntersuchungen im großen Maßstab, was bereits deutlich macht, dass die Arbeitsphysiologen nicht nur einzelne Arbeiter oder Fabriken, sondern auch das ökonomische Ganze im Blick hatten. Das Interesse am Körper des Individuums übertrug sich gleichsam auf den Gesellschaftskörper, in zeitgenössischer Begrifflichkeit: auf den Volkskörper. ${ }^{15}$

\section{Der Weg in die Öffentlichkeit: die Ausstellung „Gesolei““}

Ohne Frage handelte es sich bei der Idee des Managements von Humanressourcen in vorbeugender Absicht zunächst um einen Expertendiskurs von Wissenschaftlern. Um auch andere Akteure zu überzeugen, wurde der Weg in die Öffentlichkeit gesucht. Insbesondere Atzler ließ keine Gelegenheit aus, mit dem gedruckten Wort für seine Konzepte zu werben. Mitte der Zwanzigerjahre bot sich ihm darüber hinaus die Gelegenheit, an einer Ausstellung mitzuwirken, die seine und vergleichbare Ideen in einem bisher nicht gekannten Maßstab propagieren sollte: an der Ausstellung „Gesolei“, die 1926 in Düsseldorf ihre Pforten öffnete. Das Kürzel Gesolei stand für Gesundheits-

14 Michael Hau, Sports in the Human Economy. “Leibesübungen”, Medicine, Psychology, and Performance Enhancement during the Weimar Republic, in: Central European History 41 (2008), S. 381-412, hier S. 385.

15 Dinçkal, Sport, S. 80. 
pflege, soziale Fürsorge und Leibesübungen. Mit ca. acht Millionen Besuchern ist die Gesolei die bis heute größte Ausstellung auf deutschem Boden gewesen. ${ }^{16}$

Das Thema der Gesolei war nicht mehr und nicht weniger als der Volkskörper in all seinen Facetten. Dies galt auch in einem politischen Sinne. Schließlich sollte die Wahl des Standorts Düsseldorf darauf verweisen, dass kurz zuvor Briten und Belgier die Kölner Zone geräumt hatten, wodurch der gleichsam verletzte deutsche Volkskörper an dieser Stelle seine ursprüngliche Gestalt wiedergewann. Ebenso wichtig war aber die Sorge um die fortwirkende Gesundheit und Leistungskraft des Volkskörpers in Anbetracht der Herausforderungen der modernen Industrie. Fast alle gesellschaftlichen Kräfte, die auf den Feldern Arbeit, Freizeit und Gesundheit tätig waren, präsentierten bei der Gesolei Antworten, mit denen sie auf diese Herausforderungen zu reagieren gedachten. Industriekonzerne und Gewerkschaften, staatliche Behörden und Sozialverbände, medizinisch-hygienische Einrichtungen waren daher ebenso vertreten wie Sportorganisationen. Sie alle einte das Interesse, Arbeitsverhältnisse, Freizeitformen, vor- und fürsorgerische Interventionen so zu gestalten, dass die werktätige Bevölkerung zur dauerhaften Wahrung eines bestimmten Leistungsniveaus befähigt wurde. Wenn alle Kräfte von Wissenschaft, Wirtschaft und Staat zusammenwirkten, war eine Bändigung des Fordismus bei gleichzeitiger Nutzung seiner Produktivität möglich. Der amerikanische Weg, so behauptete diese nationalistische Deutung, ließe sich durch eine „deutsche Form“ der Rationalisierung verbessern. ${ }^{17}$

Der durch Vorsorge gebändigte Fordismus hatte ohne Frage das Potenzial, einen Konsens zu begründen. Die Gewerkschaften erhofften sich eine Wohlstandssteigerung, ohne dass die Arbeiter am Fließband zerschunden würden; die Arbeitgeber zielten auf hohe Umsätze und Belegschaften, die dauerhaft einsatzfähig und arbeitsfrisch blieben; der Staat wiederum konnte flankierend helfen mit sozialpolitischen Maßnahmen, dem Bau von Grün- und Sportanlagen und der Förderung jener Wissenschaftler, die im Namen des Fortschritts und der Verbesserung aller menschlicher Lebensbedingungen forschten. Alles in allem schien Vorsorge eine Win-win-Situation zu schaffen, da ihre Kalküle und Konzeptionen für sämtliche Akteure ein hohes Maß an Überzeugungskraft entfalteten. Zugespitzt verkörperte die Gesolei, zeitlich nicht von ungefähr zu Beginn der sogenannten Stabilisierungsphase angesiedelt, eine positive Zukunftsvision für die Weimarer Republik als Sozialstaat, Wohlstandsgesellschaft und Ort einer verantwortlich bzw. vorsorglich gemanagten Moderne.

Die Wirkungen dieses Konzepts sind bislang kaum erforscht. Wurden die Ideen, welche die Gesolei anschaulich machte und in die Öffentlichkeit transportierte, tatsächlich umgesetzt? Um Antworten auf diese Frage zu geben, soll im Folgenden eine

16 Angela Stercken, Die Gesolei als Schaubild des Körpers. Sektionen, Überblick, in: dies./Hans Körner/Gabriele Genge (Hrsg.), Kunst, Sport und Körper, Bd. 2: 1926-2004. Methoden und Perspektiven, Weimar 2004, S. 99-123, hier S. 100.

17 Becker, Rationalisierung, S. 160-166. 
exemplarische Untersuchung zur Firma Henkel durchgeführt werden - zu einem Konzern, der mit einem großen Pavillon auf der Gesolei vertreten war, sodass man von einer grundlegend positiven Haltung gegenüber den dort propagierten Leitbildern ausgehen kann. ${ }^{18}$ Und mehr noch: die Firma Henkel bediente den Diskurs der Körper-Vorsorge sogar mit ihrem wichtigsten Produkt. Das Waschmittel Persil sorgte mit seiner Reinigungskraft für besonders hygienische Verhältnisse und ließ Krankheitserregern damit keine Chance - so versprach es zumindest die unermüdlich wiederholte Werbung. ${ }^{19}$ Zum Selbstverständnis eines solchen Unternehmens hätte es gleichsam gepasst, auch im Umgang mit Arbeit und Arbeitskraft innerhalb seiner eigenen Mauern Körper-Vorsorge zu praktizieren und an neue wissenschaftliche Erkenntnisse anzuknüpfen.

\section{Vorsorgen im Unternehmen: das Beispiel Henkel}

1876 von Fritz Henkel in Aachen gegründet, war die Firma seit 1878 in DüsseldorfHolthausen ansässig. Hier verblieb der Hauptsitz, auch wenn in den Folgejahrzehnten etliche Zweigwerke hinzukamen. Nachdem bereits 1907 mit der Herstellung des Waschmittels Persil begonnen worden war, sahen die Weimarer Jahre eine Erweiterung der Produktpalette zu den Reinigungsmitteln: 1920 wurde Ata auf den Markt gebracht, ab 1922 folgten Klebstoffe. Bei den Fertigungsmethoden und bei der Organisation der Büroarbeit folgte das Unternehmen den Rationalisierungstrends der Zwanzigerjahre.

Bei der Gesolei traten auch Henkel-Sportler auf den Plan. Es handelte sich um eine Gruppe von Mitarbeitern, die sich seit 1925 zur Sportausübung in der Freizeit traf. In erster Linie wurden Fußball und Hockey gespielt. ${ }^{20}$ Die Firmenleitung hatte zunächst keinen organisatorischen Rahmen hierfür geschaffen, nahm dieses Engagement aber wohlwollend zur Kenntnis. Es fand von Anfang an in den Werkszeitungen

18 Richard Klapheck (Hrsg.), Dokument Deutscher Kunst Düsseldorf 1926. Anlage, Bauten und Raumgestaltungen der GESOLEI, Düsseldorf 1927, Abb. 122; Arthur Schlossmann (Hrsg.), GE-SO-LEI. Grosse Ausstellung Düsseldorf 1926 für Gesundheitspflege, soziale Fürsorge und Leibesübungen, Bd. 2, Düsseldorf 1927, S. 432.

19 Die Henkel-Werkspresse feierte das Produkt als „Volkshygienemittel“. Siehe z. B. Al., Zur Einweihung der Dr. Hugo Henkel-Schwimmhalle am 4. Januar 1931, in: Blätter vom Hause 11 (1931), S. 34-40, hier S. 40.

20 Konzernarchiv Henkel K 2190: Aus den Anfängen der Leibesertüchtigung bei der Fa. Henkel u. Cie. G. m. b. H. - Düsseldorf -/Sportvereine bis 1950, Bl. 1. Am 12. Juni 1926 fand ein Fußballspiel zwischen Henkelanern und der „Werksmannschaft der Preß- und Walzwerk A. G.“, ebenfalls Düsseldorf, auf dem „Sportplatz der Gesolei“ statt. Siehe Vom Werkssport, in: Werks-Bote 1 (1926), H. 3, S. 7. 
des Konzerns, die im Folgenden als Quellengrundlage dienen, starke Beachtung. ${ }^{21}$ Damit wurden offenbar mehrere Ziele verfolgt: erstens galt es, den Einsatz der sportelnden Henkelaner zu würdigen; zweitens sollten die weniger Sportlichen aktiviert werden; drittens wurden die Verdienste der Firmenleitung um die Förderung dieses wichtigen Bereichs herausgestellt.

Die Konzernspitze nahm schon seit der Gründungsphase der Weimarer Republik für sich in Anspruch, das Wohl ihrer Arbeiter trotz oder gerade im Zuge der anstehenden Rationalisierung genau im Blick zu haben.1919 verkündeten die „Blätter vom Hause“: „Wohl hat man gegen das Taylorsystem viel einzuwenden gefunden. Man sagte, der Arbeiter werde sehr ausgebeutet, seine Gesundheit leide darunter und von dem Mehrertrage käme dem Unternehmer am meisten zu. “22 Um eben dies zu verhindern, seien Gegenmaßnahmen nötig - wie sie etwa Rubners Forschungsstelle in Berlin eingeleitet hätte. „Das deutsche Institut für Arbeitsphysiologie will nun untersuchen“, führt der Artikel weiter aus, „was uns im gesamten vorwärts bringen kann: ,Wahrnehmungsfähigkeit, Schulung des Willens, Wohnräume, Arbeiterräume, Beschaffenheit der Luft, Ernährungszustände, Ueberarbeitung, Ermüdung usw. ' Das Institut will eine neue wissenschaftliche Arbeitsorganisation schaffen, die von der Arbeit ausgeht, sie dem Wirtschaftsleben in der zweckmäßigsten Weise zu erschließen und auf die beste Güterversorgung hinzuwirken.“23 Beim Bemühen um den Fortschritt „im gesamten“ durften also auch die Belange der Arbeiter nicht zu kurz kommen, wobei neben der Entlohnung auch Arbeitszeit und Arbeitsbedingungen von Bedeutung schienen - ja sogar die privaten Lebensumstände, sofern sie die Ernährung und die Wohnräume betrafen.

Wie groß das Interesse an arbeitswissenschaftlichen Studien im Unternehmen war, belegt derselbe Artikel, indem er den von dem italienischen Physiologen Angelo Mosso (1846-1910) erfundenen Ergographen vorstellt. Mit diesem Gerät konnten Arbeitsleistungen gemessen werden - zum Beispiel in Abhängigkeit von der Gewährung oder Nicht-Gewährung von Pausen zwischen den einzelnen Arbeitsgängen: „Morso [sic] hat mit diesem Apparat viele Untersuchungen angestellt und z. B. in einem Falle festgestellt, daß eine Person, welche ein Gewicht von $6 \mathrm{~kg}$ jede Sekunde einmal hob, bereits nach 15 Muskelzusammenziehungen unfähig war, das Gewicht $\mathrm{zu}$ bewegen, es war also die in Betracht kommende Muskelgruppe erschöpft. Es war nach dem Ergebnis der Messungen der betreffenden Hubhöhen im ganzen eine Arbeit von $0,912 \mathrm{mkg}$ geleistet worden. Bei einer Pause von 2 Sekunden zwischen jeder

21 Es handelt sich um die Zeitungen „Blätter vom Hause. Halbmonatsschrift der Firma Henkel \& Cie., Düsseldorf, Fabrik chemischer Produkte“, „Henkel-Bote. Mitteilungen für die Angehörigen des Henkel-Werkes. Benrath, Reisholz und Hilden“ und „Werks-Bote. Zeitschrift für die Belegschaften der industriellen Werke von Benrath, Reisholz und Hilden“, die allesamt im Konzernarchiv Henkel AG \& Co. KGaA, Düsseldorf, aufbewahrt werden.

22 E. P., Arbeit, in: Blätter vom Hause 6 (1919), S. 392-395, hier S. 395.

23 Ebenda. 
Zusammenziehung konnten deren 18 vollführt werden. Die Gesamtarbeitsleistung stieg auf 1,08. Bei Pausen von 4 Sekunden war die Anzahl der Zusammenziehungen 31, die geleistete Arbeit 1,842 mkg. Wurde das Gewicht nur alle 10 Sekunden einmal gehoben, so kam eine Ermüdungserscheinung überhaupt nicht vor. Solche Untersuchungen sind für das Maß und Tempo der Arbeit von größter Bedeutung.“24

Diese Erkenntnisse ließen sich vom einzelnen Arbeitsvorgang auf die Lebensarbeit hochrechnen. Ein weiterer Artikel aus demselben Jahr stellte kategorisch fest: „Die Firmen und Werke würden sich die Arbeitskraft ihrer Angestelltenschaft weit länger erhalten, wenn sie sich von großzügigeren Anschauungen über die Arbeitszeit leiten ließen. “25 Um der Ermüdung vorzubeugen, seien Unterbrechungen des Werkens notwendig; am besten würden sie genutzt, indem die Arbeiter und Angestellten sich der körperlichen Ertüchtigung verschrieben. Die „vom Geist der Zeit erfüllten Werke und Firmen haben darum in jeder Hinsicht vorgebeugt, wenn sie ihren Beamten [...] Gelegenheit geben, sich mehr mit der Körperpflege zu beschäftigen“. ${ }^{26}$ Die berufstätigen Menschen, „welche Sport betreiben, [...] stählen ihre Körper nach des Tages Lasten und Mühen auf von Licht und Sonne überfluteten Sportplätzen“. ${ }^{27}$ Vom Ergebnis profitieren nicht nur Arbeitgeber und -nehmer, sondern auch die Allgemeinheit: „Sportsleute und Turner sind meist abgehärtete Naturen, die nicht so leicht eine Krankheit befällt. Die Firmen werden dadurch weniger Arbeitsausfälle durch Krankheit zu beklagen haben. Auch die Krankenkassen haben davon die Nutznießung, die dadurch weniger in Anspruch genommen werden. Ebenso wird die Belegschaftsfrequenz der Krankenhäuser vermindert.“28

In den ersten Jahren nach dem Ersten Weltkrieg wurde das Sporttreiben der Belegschaften noch in einem Irgendwo jenseits der Werkstore verortet. Oft war sogar nur allgemein von körperlicher Bewegung die Rede, ohne dass Sport oder Turnen im engeren Sinne gemeint waren. Unter dem Titel „Sitzende Lebensweise“ zitierten die „Blätter vom Hause“ beispielsweise eine Stellungnahme, die den weiblichen Angestellten riet, vor dem Gang zur Arbeit, der nach Möglichkeit zu Fuß zurückgelegt werden sollte, bereits das Schlafzimmer aufzuräumen, denn ,die bei dieser Beschäftigung ausgelöste Muskeltätigkeit ist außerordentlich gesund“. ${ }^{29}$ Grundsätzlich gelte: Auch wenn ,unsere hygienischen Einrichtungen heute so gut wie möglich sind“, auch wenn die „Arbeitsräume und Werkstätten hell und gut gelüftet sind - die Gesund-

24 Ebenda, S. $393 \mathrm{f}$.

25 Ungeteilte Arbeitszeit und Körperpflege, in: Blätter vom Hause 6 (1919), S. 322 f., hier S. 323.

26 Ebenda, S. 322.

27 Ebenda.

28 Ebenda.

29 Sitzende Lebensweise, in: Blätter vom Hause 6 (1919), S. 20. 
heit kommt doch zu kurz“. ${ }^{30}$ Deswegen sollten die „freien Stunden am Tag“ und der Sonntag „soviel wie nur möglich im Freien verbracht werden“. ${ }^{31}$

Solche Aufforderungen, sich zu Hause, auf dem Weg zur Arbeit oder im Freien körperlich zu betätigen, mündeten, wie oben erwähnt, erst in der Mitte der Zwanzigerjahre bei einigen Mitgliedern der Belegschaft in eine konkrete Sportausübung ein. Die Grundlage, auf der dieses Engagement basieren sollte, war freilich schon 1919 benannt worden. „Sport, wenn er auch körperlich anstrengt, ist keine Arbeit“, sondern Spiel; und „Spiel ist Beschäftigung nach freier Wahl, während die Arbeit durch Notwendigkeit oder Pflicht oder Willen eines andern auferlegt wird“. ${ }^{32}$ Niemand, der bei Henkel Sport trieb, sollte dies als eine Fortsetzung seiner Arbeit missverstehen - auch wenn hier wie da Schweiß vergossen wurde. Weil der Sport freiwillig ausgeübt wurde, wirkte er völlig anders auf Geist und Seele zurück. „Leibesübungen sind gesundheitserhaltend und schaffen Lebensfreude“33, wusste der „Werks-Bote“ ${ }^{\text {" }}$ u berichten, sodass sie „neue Spannkraft und Widerstandsfähigkeit für die Aufgabe des Tages“34 schufen. Für eine erfolgreiche Vorsorge, verstanden als Zusammenspiel von Energieaufbau und -abbau, mussten demnach nicht nur körperliche, sondern ebenso psychische Faktoren berücksichtigt werden.

Im Gesolei-Jahr 1926 und im Folgejahr widmete die Henkel-Presse dem gesamten Themenkomplex der Rationalisierung erhöhte Aufmerksamkeit. Unter den Überschriften „Das Problem der Industriearbeit“ und „Rationalisierung“ wurde noch einmal betont, dass Rationalisierungen unumgänglich seien, weil sie die Produkte verbilligten und dadurch für mehr Wohlstand sorgten. ${ }^{35}$ Der erstgenannte Artikel führte noch weitere Gedanken ins Feld. Die Rationalisierung solle mit einer neuen Bewertung des Arbeiters und seiner Bedürfnisse einhergehen: „Dies Arbeitsdasein ihnen $\mathrm{zu}$ erleichtern, tut eins vor allem not: Die rückhaltlose Anerkennung, daß Arbeitgeber und Arbeitnehmer in einem freien Arbeits- und Rechtsverhältnis zueinander stehen. Beiden sind Rechte und Pflichten auferlegt, die mit Wohltätigkeit im alten Sinne des patriarchalischen Verhältnisses nichts mehr zu tun haben. Aus dem ,Industrieuntertan` von einst soll werden der ,Industriebürger von heute. Schutz der Gesundheit, Erhaltung der Arbeitsfähigkeit und -frische, Verhütung von Unfällen, genügende Erholungspausen, Arbeitszeit ohne dauernde Überspannung der Kräfte, Rat und rasche Hilfe in Familiennot müssen ihm gesichert sein. Sport und Spiel im Freien dienen seiner Erholung. “36 Der Rollenwandel des Arbeiters vom „Industrieun-

\section{Ebenda.}

31 Ebenda.

32 E. P., Arbeit, in: Blätter vom Hause 6 (1919), S. 392-395, hier S. 392.

33 Ge-So-Lei, in: Werks-Bote 1 (1926), H. 1, S. 3 f., hier S. 4.

34 Von der Sportbewegung der Preß- und Walzwerk A. G., in: Werks-Bote 1 (1926), H. 9, S. 7 f., hier S. 7. 35 Benser, Das Problem der Industriearbeit, in: Werks-Bote 1 (1926), H. 11, S. 3 f.; Rationalisierung, in: Werks-Bote 2 (1927), H. 6, S. $1 \mathrm{f}$.

36 Benser, Das Problem der Industriearbeit, in: Werks-Bote 1 (1926), H. 11, S. 3 f., hier S. 4. 
tertan“ zum „Industriebürger“ verband sich mit zahlreichen Rechten, zu denen auch die nachhaltige Bewirtschaftung der Arbeitskraft gehört; „Sport und Spiel im Freien“ wurden im selben Atemzug mit Unfallverhütung und „Hilfe in Familiennot“ genannt. Dabei blieb allerdings vage, ob mit diesen Rechten nicht auch Pflichten einhergingen. In einem langen Artikel zur Gesolei heißt es, der Arbeiter dürfe das „Lebensgut“ seiner Gesundheit nicht mit Ausschweifungen vergeuden; ein exzessives Wochenende könne mehr „Gesundheitsgut“ kosten, „wie in monatelanger schwerster Arbeit vertan werden kann“. ${ }^{37}$ Gesundheit und Schaffenskraft galten also als ein „Gut“, mithin als eine Ressource, die im Prinzip quantifizierbar sei und deren Zuwächse und Abgänge verantwortlich gemanagt werden sollten. Wer dies missachtete, schade nicht nur sich selbst, sondern auch dem „Wohl der Gesamtheit“..38

Vor diesem Hintergrund wurde der Fordismus ambivalent beurteilt. Seine Verbesserungen im Bereich der Produktionstechnologie und der Arbeitsorganisation, die zu einer enormen Erhöhung der Produktivität geführt hatten, wurden zwar sehr wohl anerkannt. Im Umgang mit der menschlichen Arbeitskraft erkannte man allerdings Defizite. Ein Beitrag mit dem Titel „Was will Ford?“ schildert einen fiktiven Dialog, in dem der Industriepionier seine Arbeiter fragt, ob sie mehr verdienen wollen; er sei bereit, ihnen das Doppelte zu geben - aber dafür müssten sie das Dreifache leisten. Wenn Ford die Arbeitswoche von sechs auf fünf Tage verkürze, dann nur unter der Voraussetzung, dass in fünf Tagen dasselbe geleistet werde wie vorher unter Einschluss des Samstags. Zwar betone Ford nach außen hin gerne, wie gut er seine Arbeiter behandle. Doch sei in der „Moral Fordscher Arbeit“39 die Tendenz zur Überforderung und Überanstrengung strukturell angelegt.

Neben solchen Blicken auf die USA fand in der Firma Henkel auch die Sowjetunion Beachtung. Eine Besuchsreise sowjetischer Ingenieure bildete den Aufhänger für eine Darstellung des „Werks-Boten“ über die industriellen Anstrengungen in diesem Staat. Das Bestreben der kommunistischen Wirtschaftsführer richte sich darauf, „die industrielle Erzeugung möglichst zu vervielfachen. Typisierung der Erzeugnisse und Schematisierung der Arbeit sind heute die russischen Betriebsschlagworte. “40 Dass diese Rationalisierung ,auch im kommunistischen Staats- und Wirtschaftsleben notwendig geworden ist, das haben auch die Bolschewiken erkannt““. ${ }^{41}$ Deshalb richteten sie „arbeitswissenschaftliche Lehr- und Forschungsanstalten“ ein; die „bedeutendste ist das ,Zentral-Institut der Arbeit“ in Moskau“.42 Dieses Institut stelle durch „Eignungsprüfungen, ganz nach ,kapitalistischem` Vorbilde, die Geeigneten fest und weist sie

37 Ge-So-Lei, in: Werks-Bote 1 (1926), H. 1, S. 3 f., hier S. 3.

38 Ebenda.

39 Was will Ford?, in: Werks-Bote 2 (1927), H. 7, S. 3 f., hier S. 4.

40 Arbeitsschulungsversuche in Rußland, in: Werks-Bote 2 (1927), H. 24, S. 4.

41 Ebenda.

42 Ebenda. 
den einzelnen Berufen zu. Die nach psychotechnischen Grundsätzen durchgeführte Ausbildungszeit beträgt ein halbes Jahr. Genau wie bei uns gilt der Leitsatz: Mit dem geringsten Kraftaufwand bei größter Arbeitsleistung die größten Erfolge erzielen. “43

Es ist bemerkenswert, mit welcher Nüchternheit sich die Werkszeitung über die Zustände in einem Land äußert, das dem Kapitalismus den Kampf angesagt hat. Im Wesentlichen diente das Beispiel der Sowjetunion indes dem Nachweis, dass es ohne Rationalisierung und ohne Verwissenschaftlichung des Arbeitsprozesses nicht mehr gehe - in keiner Volkswirtschaft der Welt. Auch im Bereich der „psychotechnischen Tests“ für die Arbeitseignung hielten die Sowjets mit den anderen Industrieländern Schritt. Einen Kritikpunkt brachte der „Werks-Bote“ aber dennoch an: „Ob freilich die Seele des russischen Menschen die Einbeziehung in die Rationalisierung der Wirtschaft erträgt, bleibt abzuwarten.“44 Neben aller Vorurteilslosigkeit gegenüber dem Kommunismus behielt das Klischee von der Gemütstiefe der Landsleute Dostojewskijs offenbar seine Wirkmächtigkeit.

Um es besser zu machen als die Konkurrenz in Ost und West, gab Henkel, so das Selbstbild, seinen Arbeitern hinreichende Gelegenheit zur Regeneration. 1927 schuf die Firma, nachdem es jahrelang bei Appellen geblieben war, hierfür einen institutionellen Rahmen: Eine „Sportvereinigung“ wurde aus der Taufe gehoben. Deren Anfänge waren bescheiden. Die zwölf Gründungsmitglieder wurden von einem Werkstudenten trainiert und nutzten die Plätze anderer Vereine. ${ }^{45}$ Schon bald sorgte das Unternehmen indes für Abhilfe. Es sei das ,erfolgreiche Abschneiden unserer Fußballer auf dem Gesolei-Sportplatze im Jahre 1926“ gewesen, behauptete die Werkspresse im Rückblick, das die Firma veranlasst habe, sich dieser „Bewegung anzunehmen“. ${ }^{46}$ Am 14. August 1927 wurde auf dem Werksgelände ein eigener „Sportplatz Henkel“ eingeweiht. ${ }^{47}$ Dort entfaltete sich in den Folgejahren ein reges sportliches Leben: Hatte sich die „Sportvereinigung Henkel“ zunächst nur dem Fußball und der Leichtathletik verschrieben, so umfasste sie schon bald auch eine Tennisabteilung (gegründet 1929), eine Schwimm- (1930), Handball- (1931), Turn- (1931), Kanu- (1932), Rhönrad- (1932), Ruder- (1932) und eine Schießsportabteilung (1934). ${ }^{48}$ Hinzu kamen die Pflege des

\section{Ebenda.}

44 Ebenda.

45 Konzernarchiv Henkel K 2190: Aus den Anfängen der Leibesertüchtigung bei der Fa. Henkel u. Cie. G. m. b. H. - Düsseldorf -/Sportvereine bis 1950, Bl. 1 und 3.

465 Jahre Henkelsport. Bericht über das Sportfest am 30. Juli 1932, in: Blätter vom Hause 12 (1932), S. 300-321, hier S. 300.

47 Die Einweihung des „Sportplatz Henkel“, in: Werks-Bote 2 (1927), H. 18, S. 5 f.

48 Siehe die maschinenschriftliche Auflistung „Sportvereine“ (Konzernarchiv Henkel K 2190: Aus den Anfängen der Leibesertüchtigung bei der Fa. Henkel u. Cie. G. m. b. H. - Düsseldorf -/Sportvereine bis 1950 , o. P.). 
Box- und Motorradsports, des Wanderns und des Hockeyspiels. ${ }^{49}$ Insgesamt verzeichnete die SV Henkel im Jahre 1928232 Mitglieder; 1931 waren es 550, bevor 1932 die Zahl von 700 erreicht wurde..$^{50}$ Aus der Korrelation mit der Belegschaft - 4818 Personen im Jahr $1932^{51}$ - ergibt sich, dass bis zum Ende der Weimarer Republik rund ein Siebtel der Henkelaner für den Sport gewonnen werden konnte.

Die Frage der Arbeitseignung, die der Artikel über die Sowjetunion ansprach, wurde im Übrigen auch bei Henkel diskutiert. Der „Werks-Bote“ versuchte seine Leser von der traditionellen Auffassung abzubringen, jeder Mensch solle seinen Beruf nach seiner individuellen Neigung wählen. Vorlieben, die sich in jungen Jahren bemerkbar machten, dürften keineswegs zur einzigen Grundlage für eine Entscheidung werden, bei der viele Faktoren zu berücksichtigen seien. „Der eine Junge hat Neigung zum Basteln“, veranschaulichte das Blatt seine Überlegungen, „der andere zum Lesen, Rechnen, Auswendiglernen, der dritte zum Hämmern und so weiter. Und dennoch ist es eine große Frage, ob der erste zum Mechaniker oder Elektriker, der andere zum Studium, der dritte zum Schmied, Schlosser oder Werkzeugmacher geeignet ist. Auf die Eignung zum Berufe kommt es aber an, nicht auf die Neigung!“52 Um diese Eignung festzustellen, solle man sich an die „Berufsberatung des zuständigen Arbeitsamts“"53 wenden.

Inwieweit an den Arbeitsämtern des Jahres 1928 bereits arbeitswissenschaftliche Methoden zur Feststellung der Berufseignung angewendet wurden, ließ der Artikel offen. In der Forschung jedenfalls widme man sich diesem Problem schon seit längerem, ließen die „Blätter vom Hause“ die Belegschaft wissen: „Es gibt heute eine besondere Wissenschaft der Arbeitsphysiologie. Diese wendet die Methoden der experimentellen Physiologie und Psychologie an, um die Arbeitsleistungen zu untersuchen, die für die einzelnen Berufszweige geeigneten Arbeiter auszuwählen, die Gesundheit der Arbeiter zu erhalten und zu fördern. Es gibt in Berlin ein Institut für Arbeitsphysiologie, das den Arbeitsvorgang und die Arbeitsermüdung messend verfolgt. “54 Der an diesem Institut eingeschlagene Weg orientiere sich an den Erkenntnissen ,unserer besten Naturforscher“"55; einer von ihnen, Wilhelm Ostwald ${ }^{56}$, habe den Satz geprägt:

49 Andreas Luh, Betriebssport zwischen Arbeitgeberinteressen und Arbeitnehmerbedürfnissen Eine historische Analyse vom Kaiserreich bis zur Gegenwart, Aachen 1998, S. 126.

50 Ebenda.

51 Wilfried Feldenkirchen/Susanne Hilger, Menschen und Marken. 125 Jahre Henkel 1876-2001, Düsseldorf 2001, S. 85.

52 Zur Berufswahl, in: Werks-Bote 3 (1928), H. 5, S. 5. Hervorhebung im Original.

53 Ebenda.

54 Otto Conrad, Kraftvergeudung und Kraftsteigerung, in: Blätter vom Hause 8 (1928), S.172f., hier S. 173.

55 Ebenda, S. 172.

56 Wilhelm Ostwald (1853-1932), Chemiker und Naturphilosoph. 
„Vergeude keine Energie, verwerte sie!“57 - einen Satz, den er selbst als den „energetischen Imperativ“ ${ }^{58}$ bezeichne, und den, so forderte die Werkszeitung, jeder einzelne Mensch verinnerlichen müsse: „Unser gesamtes Handeln sollte von dem energetischen Imperativ bestimmt werden. “59

Nur drei Monate später druckte der „Werks-Bote“ einen Text von Carl Diem (1882-1962) ab, Prorektor und faktischer Leiter der Deutschen Hochschule für Leibesübungen, die seit 1921 mit dem KWIfA kooperierte. ${ }^{60}$ Aufhänger für Diems Gastbeitrag waren die soeben beendeten Olympischen Spiele von 1928 in Amsterdam. Sie inspirierten den Sportwissenschaftler und -funktionär zu Reflexionen über den Stellenwert, den der Sport grundsätzlich im Leben des Menschen erhalten dürfe. Die „Verbissenheit“, die in Amsterdam teils zu Tage getreten sei, wäre völlig unangebracht: „Der Sport hat nur solange sein Recht und seinen Nutzen, solange er heitere Unterhaltung der freien Stunden bleibt“. Insofern gelte der Grundsatz: „Den Charakter fröhlichen Zeitvertreibens wollen wir wieder zu gewinnen suchen“. ${ }^{61}$ Auch für die Regeneration des werktätigen Menschen, meinten die Arbeitsphysiologen, konnte der Sport nur in dieser Form nützlich sein - Diems Stellungnahme passte sich exakt in das bei Henkel geltende Sportkonzept ein. ${ }^{62}$

Während auf der einen Seite also ein energetischer Imperativ gelten sollte, der den Arbeiter durchaus mit einer gewissen Verbindlichkeit zur Regeneration seiner Kräfte anhielt, wurde auf der anderen Seite betont, die Motivation zum Sport müsse sich aus der Freude an der Sache selbst ergeben, sie dürfe das Spielerische und das Moment der Freiwilligkeit niemals verlieren. Zwischen diesen beiden Polen bestand ein Spannungsverhältnis. Die Werkspresse, so ließe sich zuspitzen, überließ zwar dem Werktätigen die Entscheidung. Gleichwohl lieferte sie ständig Argumente für die Sportausübung - der Werktätige sollte letztlich dazu gebracht werden, aus eigener Einsicht das Gewünschte zu tun. Im Grunde lag damit ein Paradoxon vor: Die Vorsorge funktionierte nur, wenn sie auf freiwilliger Basis erfolgte und Freude bereitete. Was sollte man also tun, wenn das Vorsorgehandeln aus Nachlässigkeit versäumt oder gar vorsätzlich verweigert wurde?

57 Conrad, Kraftvergeudung und Kraftsteigerung, S. 172.

58 Ebenda.

59 Ebenda, S. 173.

60 Zu Diem siehe allgemein Frank Becker, Den Sport gestalten. Carl Diems Leben (1882-1962), Duisburg ${ }^{2} 2013$.

61 Carl Diem, Ein Schlußwort zur Olympiade, in: Werks-Bote 3 (1928), H. 19, S. 7.

62 So wurde bei Henkel auch der von der Deutschen Hochschule für Leibesübungen produzierte Film „Der neue Mensch“ gezeigt, der die Arbeit der Hochschule darstellte und für den Sport werben sollte. Die beiden Aufführungen am 22. und 25. August 1930 im Gesoleisaal des Henkelwerks wurden jeweils mit Vorträgen eingeleitet, die den Nutzen des Sports für die arbeitende Bevölkerung zusätzlich akzentuierten. Die beabsichtigte Wirkung blieb nicht aus: In der Folge gab es viele Neuanmeldungen bei der SV Henkel, von denen besonders die Leichtathletikabteilung profitierte. Siehe „Unsere Sportabteilung“, in: Blätter vom Hause 10 (1930), S. 395. 
Ein weiteres Argument für die Sportausübung war die Absenkung des Unfallrisikos. Oft war es der Konzentrationsabfall, der zu Fehlern und damit zu Unfällen führte; der Konzentrationsabfall aber resultierte zumeist aus Ermüdung. Ein gut trainierter Werktätiger würde hingegen auch an langen Arbeitstagen konzentriert bleiben. Zudem förderte der Sport Reaktionsschnelligkeit und Geistesgegenwart, zwei Eigenschaften, die ebenfalls Unfälle verhüteten. Grundsätzlich galt auch hier, wie ein Henkel-Betriebsarzt unter dem Vortragstitel „Durch Leibesübungen zur Unfallsicherheit“ feststellte, dass die „Belegschaft sich dazu durchringen [müsse], die Unfallverhütung als ihre ureigenste [sic] Angelegenheit zu betrachten“. ${ }^{63}$ Die Vorsorge im Hinblick auf Verletzungsgefahren gehört zu den zentralen präventiven Praktiken in der Geschichte der Industriearbeit; auch hierzu leistete der Sport einen wichtigen Beitrag.

Unversehrtheit, Gesundheit und Arbeitsfrische waren nicht nur für den einzelnen von Bedeutung, sondern in einem größeren Zusammenhang zu sehen. 1928 tauchte in der Werkspresse zum ersten Mal das Argument von der zunehmenden wechselseitigen Abhängigkeit im Prozess des industriellen Fortschritts auf. Die Rationalisierung treibe die Spezialisierung voran, was die wechselseitige Abhängigkeit der Menschen massiv erhöhe. Wo die „Arbeitsteilung weitestgehend durchgeführt ist, ist jeder auf das Werk und das Können seiner Mitmenschen angewiesen“. ${ }^{64}$ Nur gemeinsam könne man noch etwas vollbringen: „Jede Arbeit, auch die geringste Teilarbeit, mag sie gelernt oder nicht gelernt sein, ist daher Dienst an der Gesamtheit“. ${ }^{65}$ Umgekehrt galt aber auch: Jeder Ausfall eines einzelnen Werktätigen beeinträchtigte das Ganze, oder, bildlich wie wörtlich gesprochen, drohte die Fließbänder zu stoppen. Mit „Spiel und Sport zur Unterhaltung und Erholung nach vollbrachter Arbeit" ${ }^{66}$ sollte jedermann dieser besonderen Verantwortung gerecht werden.

\section{Vorbeugen für die „Volksgemeinschaft“}

Für die „Gesamtheit“, deren Interessen es zu berücksichtigen gelte, wurde in der Werkspresse seit den späten Zwanzigerjahren immer häufiger der Begriff der „Volksgemeinschaft“ verwendet. ${ }^{67}$ Dies dürfte auf den Einfluss des im Oktober 1925 gegrün-

63 Medizinalrat Dr. Wex, Durch Leibesübungen zur Unfallsicherheit, zitiert nach [K.] Holtk[amp], Die Abschlußfeier des Wettbewerbes zur Unfallverhütung 1932, in: Blätter vom Hause 12 (1932), S. 117. 64 Seidel, Vom Segen der Arbeit, in: Werks-Bote 3 (1928), H. 5, S. 3.

65 Ebenda.

66 Ebenda.

67 Jos. Kerzmann, Wege zu Kraft und Schönheit?, in: Blätter vom Hause 8 (1928), S. 365; „Unsere Sportabteilung“, in: Blätter vom Hause 10 (1930), S. 395. Vom „Volksganzen“ ist die Rede in Al., Füge Du, Arbeit, Hand in Hand, Herzen zu Herzen, in: Blätter vom Hause 10 (1930), S. 206-210, hier S. 210; von der „Werksgemeinschaft“ in [ ] Thierbach, Zum großen Henkel-Sportfest 1932, in: Blätter vom Hause 12 (1932), S. 254. Eine Brücke von den Werks- zu den Volksangehörigen schlägt 5 Jahre Hen- 
deten Deutschen Instituts für technische Arbeitsschulung (DINTA) zurückzuführen sein, ${ }^{68}$ hinter dem zahlreiche Industrielle aus der Rhein-Ruhr-Region standen. Das Anliegen des Instituts bestand weniger darin, selbst arbeitswissenschaftliche Forschung zu betreiben - insofern war es kein Konkurrenzunternehmen zum KWIfA. Vielmehr zielte es auf eine Implementierung arbeitswissenschaftlicher Erkenntnisse und konservativer Ordnungsmodelle in der deutschen Industrie. Gefördert werden sollte ein neuer Typus des Facharbeiters, der einen bürgerlichen Lebensstil pflegte und seine berufliche Tätigkeit so verstand, dass er Seite an Seite mit dem Arbeitgeber seinen Dienst an der Nation bzw. an der „Volksgemeinschaft“ verrichtete. Um diesen Arbeitertypus heranzubilden, förderte das DINTA die Einrichtung von Lehrwerkstätten. Dort wurden die Lehrlinge aufgrund psychotechnischer Eignungsprüfungen aufgenommen; Sport war fester Bestandteil des Ausbildungsprogramms. ${ }^{69}$

Die Rede von der „Volksgemeinschaft“, in deren Dienst, so die Henkelpresse, der Werkssport stehe, wirft natürlich die Frage auf, wie es um die Berücksichtigung der einzelnen Teile des „Volkes“ bei der SV Henkel stand. Bei ihrer Gründung 1927 befanden sich praktisch nur Angestellte in ihren Reihen; erst mit zwei- bis dreijähriger Verspätung kam eine größere Zahl von Arbeitern hinzu. Damit war aber keineswegs eine Situation erreicht, in der es zu einer Verschmelzung beider Gruppen gekommen wäre. Vielmehr ordneten sich diese unterschiedlichen Abteilungen zu. Die Hockey-, Tennis-, Wander- und Leichtathletikabteilung blieben nahezu reine Angestelltenabteilungen, während die Arbeiterschaft in der Fußball-, Handball- und Schwimmabteilung den Ton angab. ${ }^{70}$ Auf diese Grenzziehungen legten offenbar Teile der Belegschaft großen Wert. In einem Brief des Box-Obmanns an den Vorstand der SV Henkel hieß es über den „Geist“, der in der von Angestellten beherrschten Wanderabteilung herrschte: „Stellen Sie sich einmal vor, es würden zu den Wanderungen plötzlich einige Arbei-

kelsport. Bericht über das Sportfest am 30. Juli 1932, in: Blätter vom Hause 12 (1932), S. 300-321, hier S. 305.

68 Dieser Einfluss manifestierte sich in einer ganzen Serie von Artikeln in der Werkspresse im Jahr 1927. Siehe z. B. eine Schau von Arbeiten aus rheinisch-westfälischen Dintalehrwerkstätten auf der Ausstellung von Lehrlingsarbeiten der Handelskammer Düsseldorf, in: Werks-Bote 2 (1927), H. 6, S. 6; Drescher, Einweihung der Genossenschafts-Lehrwerkstatt in Benrath a. Rhein, in: Werks-Bote 2 (1927), H. 8, S. 5; Das Deutsche Institut für technische Arbeitsschulung. Die neue kommende Facharbeiterausbildung, in: Werks-Bote 2 (1927), H. 18, S. 3 f.

69 Mary Nolan, Das Deutsche Institut für technische Arbeitsschulung und die Schaffung des „neuen“ Arbeiters, in: Dagmar Reese/Eve Rosenhaft/Carola Sachse (Hrsg.), Rationale Beziehungen? Geschlechterverhältnisse im Rationalisierungsprozess, Frankfurt a.M. 1993, S.189-221; Sebastian Fasbender, Zwischen Arbeitersport und Arbeitssport. Werksport an Rhein und Ruhr 1921-1938, Göttingen 1997, S. 101-143; ders., Ein Trust des Sports. Die Arbeitsgemeinschaft der Werksportvereine der Vereinigte Stahlwerke AG, in: Gertrud Pfister (Hrsg.), Zwischen Arbeitnehmerinteressen und Unternehmenspolitik. Zur Geschichte des Betriebssports in Deutschland, Sankt Augustin 1999, S. 45-61, hier S. 48-50.

70 Luh, Betriebssport, S. 144. 
ter und Arbeiterinnen erscheinen? Bestimmt geht dann am drauffolgenden Samstag der Gruppenführer dieser Abteilung allein auf Wanderschaft. “71 Der Vergemeinschaftungseffekt bestand also nur darin, dass sich alle an irgendeinem Platz innerhalb der SV Henkel dem Sport widmeten und damit dem Vorsorgeprinzip huldigten - aber sie taten dies doch in unterschiedlicher Weise. Die „Volksgemeinschaft“ blieb sozial fragmentiert.

Eine andere Frage, die sich in diesem Zusammenhang stellt, bezieht sich auf die Mobilisierung der Geschlechter. Wurden Männer und Frauen gleichermaßen für die Vorsorge für ihre Arbeitskraft aktiv? Auch hier klafften Anspruch und Wirklichkeit auseinander. Die Henkelpresse bedauerte regelmäßig, dass die weibliche Belegschaft vom Sportangebot des Unternehmens zu wenig Gebrauch mache. ${ }^{72}$ Bemerkenswert ist ein Artikel von 1928, der den „Frauensport in Amerika“ behandelt. Die Darstellung folgt dabei dem bekannten Schema: Die Errungenschaften der Amerikaner werden prinzipiell gewürdigt, gleichzeitig aber wird auf Mängel hingewiesen, die es in Deutschland zu vermeiden gelte. Insofern sei jene Sportlichkeit der Amerikanerin zu loben, die sie schon deshalb besitze, weil die Verbreitung des Ford-Wagens sie zur „sportliche[n] Selbstfahrerin“73 gemacht habe; die allgemeine Praxis ihrer Sportausübung jedoch sei problematisch: Die Amerikanerin betreibt „ihr anstrengendes, aber auch erfolgreiches Training mit einer Hingabe und Ausdauer, die den ihr nacheifernden deutschen Frauen vorläufig noch fremd ist. Die Frau, die im Erwerbsleben steht, wird übrigens offiziell zu sportlicher Tätigkeit gezwungen. In jedem größeren Geschäftshause wird die Arbeitspause allgemein mit turnerischen Übungen und Wettkämpfen auf dem Dachgarten begonnen. Das letztere ist vielleicht wegen der damit verbundenen Anstrengungen nicht gerade vernünftig, aber die Amerikaner sind nun einmal vom ,Rekordwahn“ besessen." ${ }^{74}$ Ohne den Zwang und den Rekordwahn handele es aber sich um ein sportliches Engagement, das als vorbildlich gelten könne. Da „die Amerikanerin“ in den Weimarer Jahren vielfach als Prototyp der modernen Frau galt, konnte man auf ein Nacheifern hoffen. Jedenfalls setzte die Henkelpresse auf diesen Effekt, indem sie zum Beispiel die Föne im Werksschwimmbad ausdrücklich „den Bubiköpfen der Damen“75 empfahl - die Schwimmerin wurde wie selbstverständlich mit „modernen“ Attributen versehen.

Dieses Schwimmbad bestand seit 1931. Um dem Werkssport, der seit Beginn der Weltwirtschaftskrise 1929 mit einigen finanziellen Engpässen zu kämpfen hatte ${ }^{76}$,

71 Vertraulicher Brief des Box-Obmanns der Henkel Sportvereinigung an den Vorstand des Vereins vom 26. November 1931, zitiert nach Luh, Betriebssport, S. 145.

72 So z. B. Große Ereignisse werfen ihre Schatten voraus, in: Blätter vom Hause 12 (1932), S. 224.

73 Werner Suhr, Frauensport in Amerika, in: Blätter vom Hause 9 (1929), S. 143 f., hier S. 144.

74 Ebenda.

75 Al., Zur Einweihung der Dr. Hugo Henkel-Schwimmhalle am 4. Januar 1931, in: Blätter vom Hause 11 (1931), S. 34-40, hier S. 39.

76 Luh, Betriebssport, S. 131. 
wieder Auftrieb zu geben, nahm Unternehmenschef Hugo Henkel (1881-1953) sein 25-jähriges Firmenjubiläum zum Anlass, eine neue Sportanlage zu stiften. Das Bad war nach neuesten physiologischen Erkenntnissen als Schwimm-, Luft- und Lichtbad gestaltet, das heißt, vor einer Glasfront befand sich ein Außenbereich, wo die Badenden sich, auf Liegestühlen ruhend, Luft und Sonne aussetzen konnten. Bei hinreichenden Temperaturen wurde die Glasfront geöffnet, um die Außenluft direkt in den Schwimmbereich einströmen zu lassen. Das Becken war $25 \mathrm{~m}$ lang und $10 \mathrm{~m}$ breit, erlaubte also auch Wettkampfsport. ${ }^{77}$ Möglicherweise hatte sich Hugo Henkel vom Erfolg des Schwimmbades auf der Gesolei inspirieren lassen, das allerdings zusätzlich über einen Wellenbetrieb verfügte. ${ }^{78}$ Die Bewegung im - bewegten oder unbewegten - Wasser galt jedenfalls als besonders erfrischend, stimulierend und regenerationsfördernd. Ein „Henkel-Schwimmerlied“ goss diesen Effekt sogar in Verse: „Schlägt für uns die freie Stunde/Startet uns're Schwimmerschaft/Sie tritt an zur Schwimmerrunde/Um zu holen neue Kraft.“79

In der Betonung der stimulierenden Wirkung des Schwimmens drückt sich eine Würdigung psychischer Faktoren für die Vorbeugung von Ermüdung und „Ausbrennen“ aus, die in der Werkspresse der späten Weimarer Jahre an Bedeutung gewann. Das rein mechanische Aufrechnen verbrauchter und wieder zugeführter Energie schien der Komplexität von Arbeiterpersönlichkeit und Arbeitsvorgang nicht mehr gerecht zu werden. Jeder Mensch, ließen die „Blätter vom Hause“ verlauten, müsse „unbedingt das Recht auf ein paar frohe Stunden haben“, denn ein „froher und lustiger Mensch arbeitet mit viel mehr Lust und Liebe, aus dem Frohsinn schöpft er neue Kraft für seine Tätigkeit““. ${ }^{80}$ Immer wieder wurde auch der Aufenthalt in der schönen Natur als Kraftquell gepriesen. ${ }^{81}$ Gerade angesichts der Wirtschaftskrise und der „ungewisse[n] Lage“82, die sie in politischer wie ökonomischer Hinsicht geschaffen habe, seien Frische und Optimismus unverzichtbar ${ }^{83}$ - der Sport werde in dieser Hinsicht gar zum „Daseinskampfmittel“. ${ }^{84}$

Auch Edgar Atzler, der Leiter des KWIfA, brachte sich in diese Diskussion ein. 1928/29 war sein Institut von Berlin nach Dortmund und Münster umgezogen, was

77 [K.] Ho[ltkamp], Zur Einweihung des „Dr. Hugo Henkel-Bades“ in Düsseldorf-Holthausen, in: Werks-Bote 6 (1931), H. 2, S. 4-6, hier S. 5.

78 Becker, Rationalisierung, S. 163.

79 Konzernarchiv Henkel K 2180: Schwimmbad/Carl Henkel, Henkel-Schwimmerlied.

80 Al., Revue und Hofball heut' beim Henkel-Sport, in: Blätter vom Hause 11 (1931), S. 194 f., hier S. 194.

81 So etwa von Thierbach, Schwimmende Wanderer, in: Blätter vom Hause 12 (1932), S. 158.

82 C. Wild, Ein Jahr Dr. Hugo Henkel-Schwimmhalle!, in: Blätter vom Hause 12 (1932), S. 79.

835 Jahre Henkelsport. Bericht über das Sportfest am 30. Juli 1932, in: Blätter vom Hause 12 (1932), S. 300-321, hier S. 302.

84 A. Rühling, Das Rhönrad als neuzeitliches Turngerät, in: Blätter vom Hause 12 (1932), S. 214 f., hier S. 215. 
eine intensivierte Kooperation mit der Industrie an Rhein und Ruhr zur Folge hatte. ${ }^{85}$ In einem Gastbeitrag für die „Blätter vom Hause“ räumte Atzler ein, dass - zumindest für den geistigen Arbeiter - die „Freude an der Arbeit“ ebenfalls ein wichtiges „Antriebsmittel“ sei: „Eine Arbeit geht uns um soviel leichter von der Hand, je interessanter sie uns vorkommt. Eine langweilige Arbeit dagegen läßt in uns das Gefühl der Uebermüdung aufkommen. Wenn wir aber in diesem Zustand unsere Aufmerksamkeit und unseren Willen besonders stark anspannen müssen, um die aus einem Widerwillen gegen die Arbeit entstandenen Ermüdungsgefühle zu unterdrücken, so sehen wir ohne weiteres ein, daß langweilige Arbeit sehr viel mehr Nervenkraft erfordert, als eine Arbeit, die uns Freude macht. " ${ }^{86}$ Wenn man in das energetische Kalkül neben den körperlichen Kräften auch die Nervenkraft mit einbezog, wurden auch Arbeitsfreude und Interesse an der Arbeit zu relevanten Faktoren.

\section{Fazit}

Im Gesamtüberblick wird deutlich, dass im Umfeld des Erstens Weltkriegs eine neue Form der Körpervorsorge entstand. Sie basierte auf der Erkenntnis, dass der menschliche Körper nur über begrenzte (Arbeits-)Energie verfügt, die permanent regeneriert werden muss. Gerade im Zeichen des Fordismus, der den Arbeitern und Angestellten Höchstleistungen abverlangte, fand der „energetische Imperativ“ Beachtung, weil sonst auf Dauer ein Leistungsabfall, ja ein „Ausbrennen“ der Belegschaften zu befürchten war. Diese Erkenntnisse wurden von Arbeitswissenschaftlern, speziell Arbeitsphysiologen vorgetragen, die bei ihren Forschungen mit Sportwissenschaftlern kooperierten. Für die öffentliche Verbreitung des neuen Gedankenguts sorgte u. a. die Düsseldorfer Ausstellung Gesolei von 1926.

Die Implementierung der neuen Konzepte durch die Industrie ist weitgehend unerforscht. Am Beispiel des Unternehmens Henkel wurde hier eine erste Sondierung vorgenommen. Die Analyse der Werkspresse macht deutlich, dass Methoden zur Reduktion des Energieaufwandes im Arbeitsprozess durch Bewegungsoptimierung nur verschiedentlich angedeutet, aber kaum explizit dargestellt wurden. Umso stärkere Beachtung fand das Thema der Regeneration verbrauchter Energie. Henkel baute eine eigene Sportabteilung auf, die diesem Zweck dienen sollte. In Verbindung damit entfaltete sich ein breit gefächerter Diskurs über unterschiedliche Wege, die als Ressource interpretierte Arbeitskraft und Arbeitsfrische der Belegschaft nachhaltig zu bewahren und einem „Ausbrennen“ des Arbeiterkörpers vorzusorgen.

85 Hachtmann, Kind, S. 74.

86 Edgar Atzler, Der Kampf gegen die Ermüdung des geistigen Arbeiters, in: Blätter vom Hause 12 (1932), S. 344 f., hier S. 344. 
Seit dieser Initialzündung in der Weimarer Republik fanden und finden einschlägige Konzepte bis zur Gegenwart Beachtung. In der NS-Zeit wurden die Anstrengungen der Weimarer Zeit fortgeführt und erweitert und in Konzepte von „Volksgesundheit“ und „Rassenhygiene“ eingekleidet. Die Federführung übernahm die Organisation „Kraft durch Freude“ der „Deutschen Arbeitsfront“. Nach 1945 wurde die Sorge um die eigene Gesundheit und Leistungsfähigkeit in der Bundesrepublik stärker dem Individuum überlassen, während die DDR ihren Werktätigen Vorgaben machte, auch und gerade im Sport, der organisatorisch fast vollständig an die Berufssphäre angebunden wurde. In der jüngsten Vergangenheit hat sich das Problem des Ausbrennens und seiner Vorsorge mehr und mehr von der körperlichen auf die psychische Ebene verlagert: Die grassierenden „burn out“-Erkrankungen machen deutlich, dass auch seelische Antriebskräfte schonend bewirtschaftet werden müssen. Insofern fügt sich die ständige Beobachtung des eigenen Energieniveaus und Leistungsvermögens in der ersten Hälfte des 20. Jahrhunderts in jenes Programm der „Selbst-Optimierung“ ein, das noch heute den (westlichen) Lebensstil kennzeichnet. 


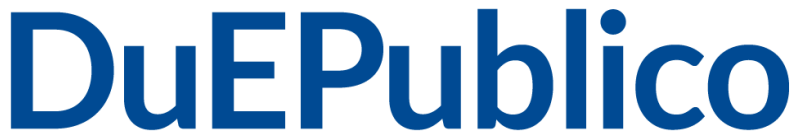

Duisburg-Essen Publications online
UNIVERSITÄT

DE $U_{S} S_{E} B_{N} U$ R G

Offen im Denken

Ub $\mid \begin{aligned} & \text { universitäts } \\ & \text { bibliothek }\end{aligned}$

Dieser Text wird über DuEPublico, dem Dokumenten- und Publikationsserver der Universität Duisburg-Essen, zur Verfügung gestellt. Die hier veröffentlichte Version der EPublikation kann von einer eventuell ebenfalls veröffentlichten Verlagsversion abweichen.

DOI: $\quad 10.1515 / 9783110529524-010$

URN: urn:nbn:de:hbz:464-20210224-113626-3

Becker, Frank: Vorsorgen oder Ausbrennen. Der Körper des Werktätigen und der „,energetische Imperativ“ in der Weimarer Republik.

In: Vorsorgen in der Moderne : Akteure, Räume und Praktiken / Hannig, Nicolai; Thießen, Malte (Hrsg.). Berlin: De Gruyter Oldenbourg, 2017 - eISBN 978-3-11-052952-4.

DOI: https://doi.org/10.1515/9783110529524, S. 191 - 212

(C) 2017 Walter de Gruyter GmbH, Berlin/Boston. Alle Rechte vorbehalten. 